in Basel and elsewhere to the recognition of three separate genes controlling the variable region of $\mathbf{I g}$. Since similar studies on the receptors of T-lymphocytes are frustrated by the difficulty of identifying, let alone purifying $T$-cell receptors, the variety of $T$-cells had to be approached by functional assays in vitro (mainly the generation of cytotoxic cells but also of helper and suppressor cells) using the improved culture methods developed in Basel to permit enumeration of single cells by limiting dilution assays. Faith and hope are now being pinned on $\mathrm{T}$-cell clones, with recognizable specificities, as a source of receptors which can be isolated and sequenced, with the aim of eventually identifying the genes controlling them and so discovering their relationship to the genes controlling Ig and the Ir region of the major histocompatibility complex.

When, in 1974, Niels Jerne put forward the network hypothesis (which states that for every specific antibody or T-cell receptor which has a distinct combining site (idiotype), the immune system is likely to contain cells able to recognize that idiotype and, in principle, to respond by making more anti-idiotype - which in turn can stimulate cells making anti-anti-idiotype etc., and so establish an infinite network of interactions), some of his colleagues put it to the test. They found that in the special circumstance when mice respond to a bacterial antigen by making antibodies with predominantly a single idiotype, specific anti-idiotype antibodies could either stimulate or suppress antibody formation against the original antigen. The network hypothesis was thus shown to have at least one real mesh; subsequent work on idiotypes has established several more, and a fair amount of the work going on in Basel (and of the more speculative essays in these volumes) is orientated towards the network hypothesis. However since the Institute has a horizontal structure, in the sense that in principle no scientist can tell any other scientist what to do, aspects of immunology which profoundly affect the practical outcome of antigenic stimulation - such as lymphocyte recirculation and the role of macrophages - are also studied there, even though Jerne thought them irrelevant to the basic questions which he hoped to see answered.

The volumes contain essays on all of these subjects, and even some rather good ones on non-specific factors, such as

The second volume of Elsevier's Dictionary of Botany, edited by Paul Macura, has recently been published by Elsevier Scientific. (For review of Vol.I, Plant Names, see Nature 284, 382; 1980. ) Volume II, General Terms, contains some 10,000 entries of English, French and German terms used in botany and associated disciplines, and includes French, German and two Russian indexes, one numerical and the other alphabetical. The book costs Df1.260, $\$ 110.75$. complement, by scientific advisers who reckoned that Jerne should recognize that they also are important. The more speculative essays, with few or even no references, are included in the first volume and the harder science in the second.

It may reasonably be asked what a collection of this nature has to offer to persons outside the charmed circle of Jerne's associates. Since the contributors felt challenged to write something original, and since free speculation was encouraged and the personalities of the authors show through in their writing, the collection provides enough stimulation to satisfy any reasonably knowledgeable immunologist. It would take a keen seeker after enlightenment to read through all of the 900 odd pages, but since the editors have grouped the essays under subject headings, selective browsing is quite possible. I would expect to recognize the striking dust jacket (by Jean Tinguely) on train and aeroplane journeys, and even at the bedside.

J.H. Humphrey is a Professor in the Department of Medicine at the Royal Postgraduate Medical School, University of London.

\title{
Down and beyond the composite particle
}

\section{M.G. Albrow}

Jets of Hadrons. By W. Hofmann. Pp.210. ISBN 3-540-10625-1/0-387-10625-1. (Springer-Verlag: 1981.) DM 62, \$28.20.

THE study of strongly interacting particles (protons, neutrons, pions etc.) and the forces between them is no longer frontier physics. We have passed beyond and down to the next layer. It has become established beyond any reasonable doubt that these socalled particles are not elementary at all, but composites of more fundamental constituents. The main effort in highenergy physics is now directed towards studying these constituents and the forces between them. A vast amount of experimental information is all consistent with a theoretical framework describing these constituents as fractionally charged quarks and antiquarks which bind together by exchanging electrically neutral gluons. These entities seem to be at the same level of elementarity as electrons, muons, neutrinos and photons, yet the latter are freely observed unlike quarks and gluons. One can do an experiment (for example scatter a high-energy muon through a known large angle on a proton) which should produce a quark with a known energy and direction. No quark is seen, always a shower of normal composite particles (hadrons) such as pions, kaons and so on. Try harder by increasing, to the limit of present accelerators, the energy of the muon or the angle of scatter, and the showers become more collimated. They are known as jets of hadrons.

Our theoretical outlook has changed so much and the amount of experimental information has grown so rapidly in recent years that Hofmann's review of the field is extremely welcome. To illustrate this rapid development most of the 400 papers in the reference list have been published in the past four years. In such a situation the author must of course take the risk that the book becomes quickly dated. If it does, I suspect that it will be due to the availability of more (and more precise) experimental data rather than to the concepts and the formalism becoming outdated.

The book "is intended to give an introductory review to the phenomenon of jets in hadronic final states..." , and the author succeeds admirably in fulfilling this intention. Starting from the simplest situation of $\mathrm{e}^{+} \mathrm{e}^{-}$annihilation (where the massive virtual photon produced by the annihilation transforms to a $q \bar{q}$-pair, which in turn materializes as two jets), he leads the reader through jets in parton models and their development as quantum chromodynamics (the theory of quark--gluon forces) to the more complicated processes of hadron-hadron collisions. A chapter on the old "uncorrelated jet model" and one on "soft" hadronic collisions are useful reminders of the importance of phase space effects and four momentum conservation, and also that while theory has a long way to go in describing the most common collisions there is encouraging progress in that direction.

It is a pity that the author was not prepared to address the question of future developments. Now that proton-antiproton collisions are being studied at CERN at energies nearly ten times higher than previously available at the ISR, and that LEP has received approval to study high-energy electron-positron collisions, a discussion of expectations for future experiments would have been a useful addition. Nonetheless, this review is sufficiently detailed and complete that theorists working in the field will find it a valuable guide to the existing experimental data, and experimentalists will appreciate the exposition of the theoretical ideas. It is, however, written at a level such that graduate students in high-energy physics should not find it too heavy-going, and it will familiarize them with what may well remain a major topic in the subject over the next decade.

M.G. Albrow, currently at CERN, is a Research Physicist at the Rutherford Appleton Laboratory, Chilton, Oxfordshire. 